Z Epileptol 2012 $25: 138-138$

DOI 10.1007/s10309-012-0246-6

C) Springer-Verlag 2012

H. Beck ${ }^{1} \cdot$ H. Lerche ${ }^{2}$

${ }^{1}$ Klinik für Epileptologie, Universität Bonn

${ }^{2}$ Hertie-Institut für klinische Hirnforschung, Tübingen

\title{
Laudatio
}

\section{Zur Verleihung der Ehrenmitgliedschaft der DGfE in Stuttgart 2012 an Herrn Prof. Dr. Uwe Heinemann}

Pathophysiologie an der Universität zu Köln und nach anderen Angeboten die Übernahme des Lehrstuhls für Neurophysiologie an der Charité in Berlin. Besonders wichtig war ihm immer die enge Kollaboration zwischen Grundlagenwissenschaften und Klinik. Er hatte deshalb zahlreiche Kliniker in seiner Gruppe zu Gast, um bei ihm klinisch orientierte Fragen mit grundlagenwissenschaftlichen Methoden zu bearbeiten. Er hat sowohl lokale Graduiertenschulen als auch ein Marie Curie-Trainingscenter mit gestaltet. Seine internationalen Aktivitäten schlossen die langjährige Tätigkeit in der Kommission für Neurobiologie und Epilepsie der ILAE sowie Advisory Boards zur European Academy of Epilepsy und den internationalen großen Kongressen mit ein.

Zudem war er ein wesentlicher Initiator der heute etablierten grundlagenwissenschaftlichen Veranstaltung der ILAE „Workshop on Neurobiology of the Epilepsies“. Seine wissenschaftliche Exzellenz und integrative Persönlichkeit wurde durch viele Preise ausgezeichnet, z. B. erhielt er zweimal den internationalen Michael-Preis, den Alfred Hauptmann-Preis, den Europäischen Epilepsie-Preis und den Milken Award der American Epilepsy Society. Auch um die Deutsche Gesellschaft für Epileptologie machte er sich sehr verdient und war von 1993 bis 1994 ihr Erster Vorsitzender.
Aus diesen Gründen freuen wir uns, Herrn Prof. Heinemann heute die Ehrenmitgliedschaft der Deutschen Gesellschaft für Epileptologie zu verleihen.

Für den Vorstand der DGfE

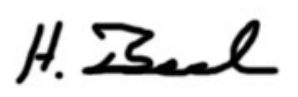

Prof. Heinz Beck

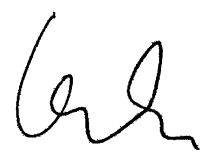

Prof. Holger Lerche 